\title{
MiRenSVM: towards better prediction of microRNA precursors using an ensemble SVM classifier with multi-loop features
}

\author{
Jiandong Ding ${ }^{1}$, Shuigeng Zhou ${ }^{1 *}$, Jihong Guan ${ }^{2^{*}}$ \\ From The $21^{\text {st }}$ International Conference on Genome Informatics (GIW2010) \\ Hangzhou, People's Republic of China. 16-18 December 2010
}

\begin{abstract}
Background: MicroRNAs (simply miRNAs) are derived from larger hairpin RNA precursors and play essential regular roles in both animals and plants. A number of computational methods for miRNA genes finding have been proposed in the past decade, yet the problem is far from being tackled, especially when considering the imbalance issue of known miRNAs and unidentified miRNAs, and the pre-miRNAs with multi-loops or higher minimum free energy (MFE). This paper presents a new computational approach, miRenSVM, for finding miRNA genes. Aiming at better prediction performance, an ensemble support vector machine (SVM) classifier is established to deal with the imbalance issue, and multi-loop features are included for identifying those pre-miRNAs with multiloops.

Results: We collected a representative dataset, which contains 697 real miRNA precursors identified by experimental procedure and other computational methods, and 5428 pseudo ones from several datasets. Experiments showed that our miRenSVM achieved a 96.5\% specificity and a 93.05\% sensitivity on the dataset. Compared with the state-of-the-art approaches, miRenSVM obtained better prediction results. We also applied our method to predict 14 Homo sapiens pre-miRNAs and 13 Anopheles gambiae pre-miRNAs that first appeared in miRBase13.0, MiRenSVM got a 100\% prediction rate. Furthermore, performance evaluation was conducted over 27 additional species in miRBase13.0, and 92.84\% (4863/5238) animal pre-miRNAs were correctly identified by miRenSVM.
\end{abstract}

Conclusion: MiRenSVM is an ensemble support vector machine (SVM) classification system for better detecting miRNA genes, especially those with multi-loop secondary structure.

\section{Background}

MicroRNAs (miRNAs) [1] are single-stranded, endogenous 22 nt small non-coding RNAs (sncRNA) that can play important regular roles in animals and plants by targeting mRNA for cleavage or post-translation repression [2]. Mature miRNAs are derived from longer precursors (pre-miRNAs), each of which can fold into a hairpin structure that contains one or two mature

\footnotetext{
* Correspondence: sgzhou@fudan.edu.cn; jhguan@tongji.edu.cn 'Shanghai Key Lab of Intelligent Information Processing, and School of Computer Science, Fudan University, Shanghai 200433, China ${ }^{2}$ Department of Computer Science and Technology, Tongji University, Shanghai 201804, China

Full list of author information is available at the end of the article
}

miRNAs in either or both its arms. Accordingly, miRNA biogenesis is highly regulated, controlled at both transcriptional and post-transcriptional levels [3], and overexpression and underexpression of miRNAs are linked to various human diseases, particularly cancers $[4,5]$.

MiRNAs are always located in the introns of proteincoding genes [6], introns and exons of non-coding genes [7]. In mammalian genomes, it is also possible to find miRNAs in repetitive regions, and some studies suggest that transposable elements may be involved in the creation of new miRNAs [8]. MiRNA biogenesis in animals contains two steps [2]. In the first step, the primary miRNA (pri-miRNA), which is several hundred nucleotides long, is 
processed in the nucleus by a multiprotein complex containing an enzyme called Drosha to produce the $\sim 70 \mathrm{nt}$ long miRNA stem-loop precursor (pre-miRNA), which is then exported to the cytoplasm. In the cytoplasm, the second step takes place where the pre-miRNA matures into a 22nt long miRNA:miRNA* duplex, with each strand originating from opposite arms of the stem-loop [9]. Then, the miRNA strand of the miRNA:miRNA* duplex is loaded into a ribonucleoprotein complex known as the miRNA-induced silencing complex (miRISC). Until recently, the miRNA* was thought to be peeled away and degraded. However, some studies indicate that miRNA* is also sorted into Argonauts and might have a regular function in Drosophila melanogaster $[10,11]$.

Identification of miRNA genes is an eminent and challenging problem towards the understanding of post-transcriptional gene regulation. The short length of miRNAs and their ability to act redundantly or to have only a subtle phonotypical impact impose a limitation to the use of mutagenesis and other conventional genetics techniques [12]. Direct cloning is the initial choice, but only abundant miRNA genes can be easily detected. Since not all miRNAs are well expressed in many tissues, miRNAs that have very low expression levels or that are expressed tissue-specifically possibly can not be detected, and recently research suggests that lowly expressed Human miRNA genes evolve rapidly [13]. This situation is partially mitigated by the deep-sequencing techniques that nevertheless require extensive computational analysis to distinguish miRNAs from other small non-coding RNAs of the same size [14]. Therefore, computational approaches are essential for miRNA gene finding in sequenced genomes.

In these years, large-scale computational approaches have been developed, such as filter-based approaches $[6,15]$, homology-based research [16,17], mixed approaches [14,18], and machine learning methods. Filter-based approaches (e.g. MirScan, mirSeeker), focusing on identifying high-quality sets of conserved miRNA candidates, are able to recover a substantial part of the known miRNAs. However, they are critically dependent on conservation criteria to obtain reasonable specificity. Homology-based approaches (e.g. ERPIN, MiRAlign) rely exclusively either on sequence conservation or structure conservation so that lineage- or species-specific miRNA genes may escape the detection. In fact, many miRNA gene prediction approaches incorporate a homology search as part of their protocols, in addition to the ordinary search for orthologous. Mixed approaches (e.g. PalGrade, miRDeep) combine experimental with computational procedures in order to identify a wider range of miRNAs. As mentioned above, experimental approaches cannot easily detect low-expression or tissue-specific miRNAs.

The most popular computational miRNA gene finding methods are machine learning based approaches. Most of them share the same overall strategy but use different approaches to identify good stem-loop candidates, since they all try to generalize a positive set of already known miRNAs and a negative set of stem-loops that are not pre-miRNAs [19]. Several machine learning methods have been proposed to tackle the problem of identifying miRNA genes. SVM is a popular framework used to learn the distinctive characteristics of miRNAs. There are other machine learning methods that employ techniques such as HMM (Hidden Markov Model) [20,21], Random Forests [22], Naïve Bayes classifier [23], and Random walk algorithm [24] etc. Most approaches use sets of features including sequence conservation [25-27], topological properties $[26,28]$, thermodynamic stability $[26,27]$, and some other properties like entropy measures [27].

However, there are two major drawbacks with the existing machine learning based miRNAs identification approaches. One drawback is raised by the imbalance of positive and negative examples used. Since the real number of miRNAs in any given genome is still an open problem, it is assumed that there is a very few miRNA precursors in any randomly chosen stem-loop extracted from the genome. Positive examples are usually selected from miRNAs identified by experimental procedures or other computational methods. And the number of positive examples we can obtain is substantially smaller than that of negative examples. The imbalance issue between positive and negative examples can greatly degrade the performance of current machine learning approaches. Certainly, with a growing number of miRNAs being identified, we can expect an increasingly better performance from these methods. The other drawback lies in the fact that most existing machine learning based methods [23-25] make a few structural assumptions concerning stem length, loop size and number, as well as minimum free energy (MFE). Therefore, sequences with multi-branched loops secondary structure or MFE higher than $-16 \mathrm{kal} / \mathrm{mol}$ possibly can not be predicted by those methods, which subsequently degrade the prediction performance. We have investigated Homo sapiens miRNAs in miRBase [29], and found that there are an increasing number of pre-miRNAs, which do not satisfy the above-mentioned assumptions (see Table S1 and $\mathrm{S} 2$ in the Additional file 1 for detail).

In this paper, we still treat the miRNA gene finding problem as a classification problem, and develop a powerful classification system, named miRenSVM, to overcome the two drawbacks mentioned above. On one 
hand, miRenSVM uses ensemble learning to deal with the imbalance issue; On the other hand, in addition to the features exploited by the existing methods, miRenSVM further includes the multi-loop features in its classifiers, and $F$-score is used to select final classification features. As a result, miRenSVM can achieve better performance than the existing methods.

In summary, miRenSVM distinct itself from the existing methods at least in three aspects: (1) Lower expression and tissue-specific miRNAs can be easily identified since different types of features are use. (2) Due to using ensemble SVM classifiers, both positive and negative examples can be exploited as many as possible. (3) No structural assumption for miRNA candidates is made. Particularly, multi-loop features are considered.

\section{Results}

Results of different features sets

We used 65 local and global features that are subsumed into three groups, which capture miRNA's sequence, secondary structure and thermodynamic properties respectively. In this section, we used single SVM classifier to check how different feature sets impact classification performance.

First, we trained a single SVM classifier with the entire training dataset to examine prediction performance by using each of the three features group separately. The classification performance is evaluated by the outer 3fold cross validation method, which has been described in the method section. The results are listed in Table 1. Among the three feature subsets, the base pair group gets the highest SE (87.38\%), while the thermodynamic group archives the best SP (98.99\%), $\mathrm{G}_{\mathrm{m}}(92.84 \%)$ and Acc (97.59\%). The triplet elements group obtains a good SP $(98.39 \%)$, but its SE is only $74.93 \%$, which is much lower than that of the other two groups. From Table 1, we can see that: 1) Thermodynamics features are more discriminative than structure and sequence features in identifying miRNA precursors. Similar result was also obtained in [30]. 2) Base pair features are more useful in predicting real pre-miRNAs, since base pair group gets the highest sensitivity. 3) The four multi-loop features introduced in miRenSVM are effective in predicting pre-

\begin{tabular}{|c|c|c|c|c|c|}
\hline Feature Group & num & SE(\%) & SP(\%) & Gm(\%) & Acc(\%) \\
\hline triplet element & 32 & 74.93 & 98.39 & 85.87 & 95.64 \\
\hline base pair & 15 & 87.38 & 98.24 & 92.65 & 97.00 \\
\hline thermodynamics & 18 & 87.07 & 98.99 & 92.84 & 97.59 \\
\hline all features & 65 & 87.50 & 98.82 & 92.99 & 97.47 \\
\hline Selected by F-score & 32 & 87.78 & 98.88 & 93.16 & 97.58 \\
\hline
\end{tabular}

miRNAs with multi-loops, considering that nearly $84 \%$ pseudo pre-miRNAs and $4.76 \%$ real pre-miRNAs have secondary structure with multi-loops.

Second, all the 65 features were used to train a single SVM classifier with the whole training dataset, and the performance was also evaluated by the outer 3 -fold cross validation method. The results are SE (87.50\%) and $G_{m}(92.99 \%)$, which are a little better than the best results of using any individual features group. This indicates that the combination of different kinds of features can improve classification performance. The next step is to improve the prediction speed without degrading the accuracy rate. We thus considered feature selection method to select the intrinsic ones from all the 65 features. Feature selection is often applied to high dimensional data prior to classification learning. This procedure can reduce not only the cost of recognition by reducing the number of features that need to be collected, but in some cases it can also provide a better classification accuracy due to the finite sample size effect [31]. Here, we used $F$-score to select the best feature subset for our miRenSVM. This procedure is implemented by the libsvm's feature selection tool. We evaluated the effectiveness of the feature subset selected by $F$-score method by training a single SVM classifier on the entire training set, and studying the sensitivity and the number of correctly predicted miRNAs. All the results of these experiments are summarized in Table 1. As shown in Table 1, after feature selection, the classification performance becomes better.

At last, the 32 features with the largest $F$-scores were used to train the miRenSVM classifier. This feature set contains 8 features from the triplet elements group, 8 features from the base pair group and 16 features from the thermodynamic group. Experimental results show that the 32 selected features subset not only obtains the highest classification results, but also greatly reduces the outer and inner cross-validation training time taken by SVM ensembles, especially when conducting class imbalance learning experiments presented in the next section. Table 2 lists all features used in the final SVM ensembles.

\section{Results of SVM ensembles}

In this section we will present the experimental results of our miRenSVM approach. Two schemes, majority vote and mean distance (detail was delayed to the method section) were applied to aggregating the results of each sub SVM classifier. Since the ratio of negative samples to positive samples is $7.79: 1$, the cases of $k=1$, $2,3,4$ or 8 were tested, respectively. We found that sub SVM classifiers trained with negative samples which are closer to the positive samples always achieve a lower SE than the other cases. And we called these datasets 
Table 232 features selected by $F$-score

\begin{tabular}{|c|c|c|}
\hline Group & num & Feature \\
\hline triplet element & 8 & $A\left(\left(l, A_{\ldots} \ldots, U((), U(),. U \ldots, G),((, C)((, C) .(\right.\right.$ \\
\hline base pair & 8 & $d P, d P / n \_l o o p s$, Avg_bp_stem, diversity, $|A-U / L| G-C \mid L,, \%(A-U) / n \_l o o p s, \%(G-C) / n \_l o o p s$ \\
\hline thermodynamics & 16 & NEFE, MFEI ${ }_{1}, M F E I_{2}, M F E I_{3}, M F E I_{4}, d G$, Diff, Freq, Tm, dH/L, dS/L,Tm/L, $p$-value_MFE, p-value_EFE, z-score_MFE, z-score_EFE \\
\hline
\end{tabular}

"closer set". With this observation, we perform majority vote when even number of sub classifiers are employed in our miRenSVM. That is, when $k$ is even (e.g. 2 , 4 , or 8 ) and the test sample receives equal numbers of positive and negative votes, the latter half of sub SVM classifiers takes priority over the former half trained with closer sets. Here, all experiments were conducted through the outer 3 -fold cross validation method. Table 3 presents the average classification results of some SVM ensembles experiments.

As shown in Table 3, both majority vote and mean distance get a better performance than using a single SVM classifier developed with the 32 selected features $\left(\mathrm{G}_{\mathrm{m}}=93.16 \%\right)$. Compared with mean distance method, majority vote always archives higher sensitivity (SE), but its specificity (SP) is much lower, which impacts its overall accuracy (Acc). If this type of classifier is used for real-life prediction, due to its lower specificity, the chance of incorrectly predicting random sequences with stem-loop like secondary structure would be quite high. Therefore, we choose the best classifier developed under the mean distance method as the final miRenSVM classifier. The mean distance method obtains the best classification results on our dataset, that is, the highest $G_{m}$ (94.76\%) with $\mathrm{SE}=93.05 \%$ and $\mathrm{SP}=96.5 \%$, and an acceptable Acc (96.1\%). There is another reason to choose mean distance, that is efficiency. The ensemble SVM classifier predicts each test sample only one time while each test sample has to be predicted $k$ times under majority vote.

We then validated our miRenSVM on the testing dataset. This set contains 14 Homo sapiens and 13 Anopheles gambiae miRNA precursor sequences newly published in miRBase13.0. The result shows that miRenSVM obtains $100 \%$ accuracy. Particularly, 4 sequences (MI0009983, MI0009988, MI0010486, and MI0010488) in the testing set whose MFE is higher than -13.70 kal/

Table 3 Results of classifier ensembles with different aggregation methods

\begin{tabular}{lllll}
\hline Method & $\mathrm{SE}(\%)$ & $\mathrm{SP}(\%)$ & $\mathrm{Gm}(\%)$ & $\mathrm{Acc}(\%)$ \\
\hline majority vote $(k=3)$ & 97.23 & 92.10 & 94.63 & 92.70 \\
majority vote $(k=8)$ & $\mathbf{9 7 . 9 1}$ & 91.08 & 94.44 & 91.89 \\
mean distance $(k=3)$ & 93.05 & 96.50 & $\mathbf{9 4 . 7 6}$ & 96.10 \\
mean distance $(k=4)$ & 90.55 & $\mathbf{9 7 . 7 9}$ & 94.10 & $\mathbf{9 7 . 9 1}$ \\
\hline
\end{tabular}

For each aggregation method, only the best two results are presented. mol are all predicted correctly by our miRenSVM. In order to further demonstarte the advantage of the miRenSVM approach, we tested our miRenSVM on the miRBase13.0 and achieved a high sensitivity. MiRBase13.0 contains 27 animal genomes, including 5238 miRNA precursor sequences (not including $h s a$ and $a g a$ pre-miRNAs). MiRenSVM correctly classified $92.84 \%$ (4863/5238) pre-miRNAs.

\section{Results of comparison with existing methods}

We compared our approach with three existing methods that also used machine learning techniques to predict miRNA precursors [23, 25, 32] . These three compared methods include triplet-SVM, BayesMiRNAfind and microPred. The results of these methods are obtained by predicting 2060 sequences (250 real and 1810 pseudo pre-miRNAs) that have been already used in developing our MiRenSVM. This dataset contains two parts: $1 / 3$ training set (223 real and 1810 pseudo pre-miRNAs) and the smaller testing set (27 bran-new hsa and aga pre-miRNAs). The results of these experiments are illustrated in Figure 1.

triplet-SVM was proposed by Xue et al. [25] to recognize pre-miRNAs based on the triplet element structuresequence features. The method is trained on known human pre-miRNAs and obtains a high accuracy ( $90 \%)$ when applied to several other species. Unlike miRenSVM, triplet-SVM uses only structure-sequence information, and therefore can predict miRNAs quickly. However, this method is not designed to detect miRNAs with multi-loop secondary structure or miRNAs with high MFE. triplet-SVM predicts only 518 (235 real and 283 pseudo) sequences. Although it has an acceptable sensitivity $(84.68 \%)$, its specificity $(77.74 \%)$ is not comparable to ours (96.5\%).

BayesMiRNAfind was developed by Yousef et al. [23], which uses a different machine learning method, naïve Bayes classifier, to predict miRNAs conserved between human and mouse. Yousef et al. applied their method to the forward strand of the mouse genome sequence and present results for different score cut offs. BayesMiRNAfind is trained with cross-species dataset, which contains 13 different organisms. Results show that our miRenSVM detects more already known pre-miRNAs than BayesMiRNAfind: of the total 250 real pre-miRNAs, BayesMiRNAfind correctly predicts 220, while miRenSVM correctly predicts 233 . Most of the negative 


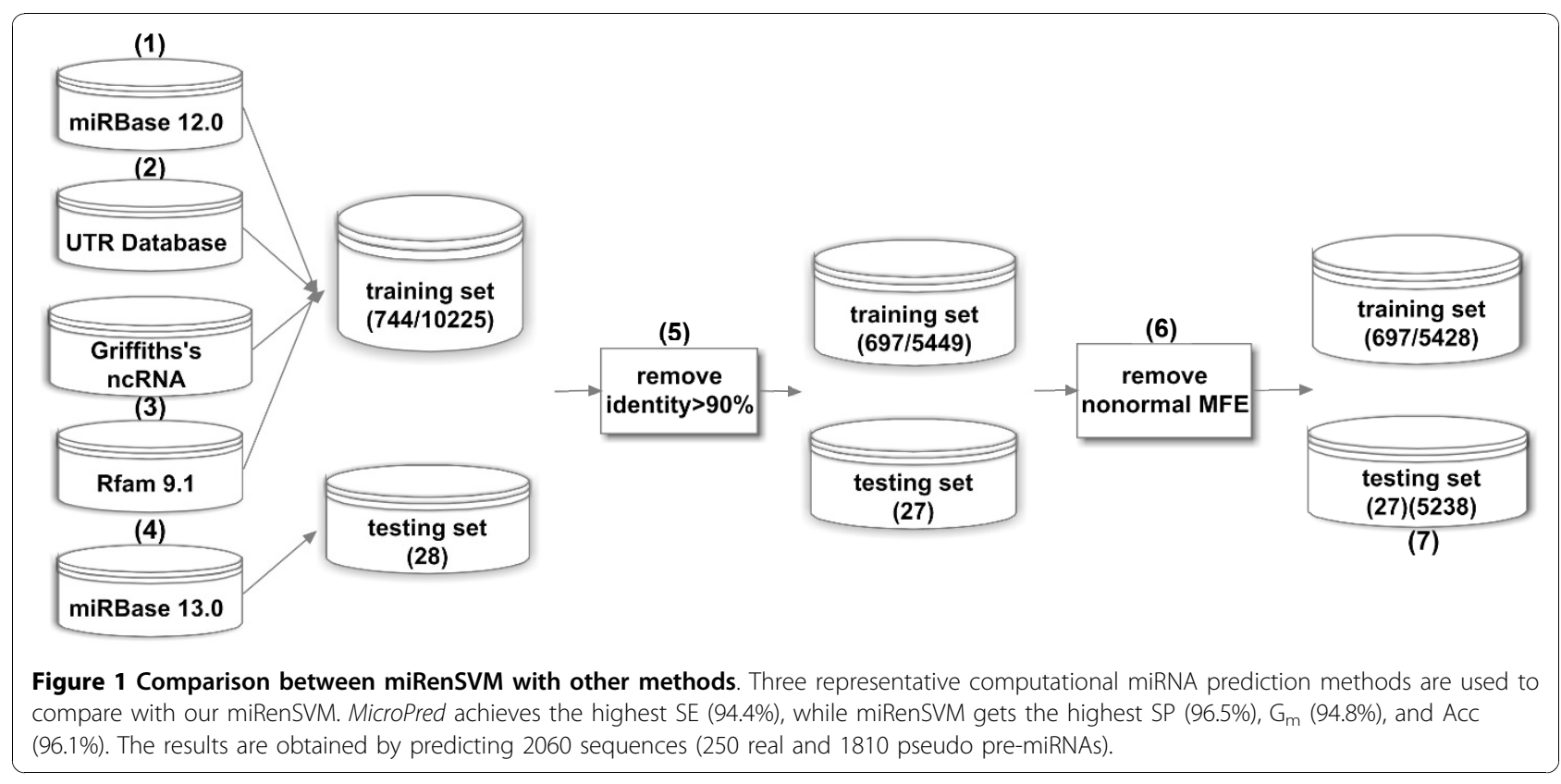

training samples ( 92\%) used in our miRenSVM are also used to train BayesMiRNAfind. BayesMiRNAfind detects 1695 out of 1810 sequences in 3'-UTRdb and Rfam, while miRenSVM finds 1746 of the same 1810 sequences, thus miRenSVM achieves a much higher specificity.

MicroPred is an SVM-based method designed recently by Rukshan and Vasile to detect human miRNA gene [34]. Like miRenSVM, microPred uses 29 different features for SVM classification, and employs SMOTE to deal with the class imbalance problem. Although the features used in microPred is a little different from that in miRenSVM, they also cover the sequence, structure and thermodynamics aspects of miRNA precursors. Also trying to improve performance with an imbalance learning method, microPred achieves a sensitivity of little higher than our method: out of the 250 known miRNAs in miRbase12.0, microPred detects 236 and we detect 233. However, microPred predicts $516 \%$ more miRNA candidates than miRenSVM (394 compared to 64). Thus, miRenSVM has a much higher specificity than microPred, although microPred specificity is estimated high. The better performance of miRenSVM is possibly due to the features used in the classification system. Considering that a large number of pseudo stem-loop sequences have secondary structure with multi-loops, microPred uses only one multi-loop relevant feature $\left(M F E I_{4}\right)$, while miRenSVM uses four $\left(\mathrm{MFEI}_{4}, d P / n_{-}\right.$loops, $\%(A-U) / n_{-}$loops, $\%(G-C) / n_{-}$loops $)$.

\section{Discussion}

The miRenSVM was first trained on Homo sapiens and Anopheles gambiae genomes, and got $93.05 \%$ sensitivity, 96.5\% specificity and $96.1 \%$ accuracy via outer 3 -fold cross validation method. We then applied it to detect new miRNAs of $h s a$ and aga genome in miRBase13.0. All 27 new pre-miRNAs were correctly detected. To further demonstrate the advantage of our approach, we tested miRenSVM on 27 additional animal genomes registered in miRBase13. Out of the 5238 animal premiRNAs across the 27 other species, miRenSVM correctly identified 4863 , i.e, the recognition rate is $92.84 \%$. The approach outperformed another recently published method [32] in detecting miRNA precursors with multibranched loops, and obtained higher and more reliable results than the existing methods $[23,25,32]$, while there is a little overlap among sets of miRNA candidates predicted by the different methods.

Since the number of possible candidate hairpins within the whole genome is very large and the number of real pre-miRNA is still small for some species, current specificity is still not satisfactory for multi-genomes applications and some false positive predictions can be produced. Finding more information to reduce the false positive rate should be further investigated. However, latest reports suggested that some human miRNA precursors have Box H/ACA snoRNA features [33]. It might be necessary for us to reconsider those previously regarded as false-positive predictions, since our dataset contains a certain amount of $h s a$ and aga snoRNAs. 


\section{Conclusion}

In this study, we presented miRenSVM, a SVM-based computational approach that detects real miRNA precursors from pseudo ones with their intrinsic features. MiRenSVM uses both global and local intrinsic features of known miRNAs as its input. Several machine learning technologies including feature selection, imbalance learning and multi-classification were applied. Our approach is more general than the existing methods, since it is not sensitive to pre-miRNA's structure and thermodynamic characteristics. And it can achieve better prediction performance than the existing methods.

\section{Methods}

\section{Dataset}

Constructing positive and negative samples sets is essential to training a machine learning classifier. It is naturally to take the already known miRNAs as the positive samples. The difficulty is to decide the best negative samples for training the classifiers. Since the number of miRNAs in a given genome is unknown [19], it is not suitable to randomly extract stem-loops sequences from the genomes. To produce high specificity in the prediction of new candidate miRNAs, the negative examples should be highly similar to the miRNA themselves. We collected negative samples in two ways: (1) Using samples from the mRNA 3'-untranslated region (3'-UTR). It has been proved that there is none predicted hsa and aga miRNA sequence in the UTRdb $[23,34]$. (2) Using ncRNA recognized so far including these miRNA from
Rfam9.1 [35] and other datasets. The resulting dataset contains two kinds of representative species, Homo sapiens ( $h s a$ ) and Anopheles gambiae (aga), both have been well studied in previous researches $[23,24,36]$. Construction of the dataset including both training and testing samples involves several steps. Figure 2 illustrates the process where each step is described as follows.

(1) $692 \mathrm{hsa}$ and 52 aga pre-miRNA sequences in miRBase12.0 were chosen to serve as the positive set.

(2) $9225 \mathrm{hsa}$ and $92 \mathrm{aga}$ 3'UTR sequences in 3'UTRdb (release 22.0) whose length ranges from 70nt and 150nt were chosen to form one part of the negative set.

(3) For $h s a$, an ncRNA dataset was already collected by Griffiths-Jones [37] that was used in [32] lately, but none sncRNA dataset of aga is available now. We selected all 256 aga ncRNA sequences in Rfam9.1, in which 68 sequences that were redundant or longer than 150 nt were removed. These sequences form another part of the negative set, which are listed in the additional file 2 .

(4) 14 hsa and 14 aga new hairpin sequences in miRBase13.0 were used to evaluate our miRenSVM system.

(5) In this step, sequences with the similarity score higher than 0.90 were removed by CD-HIT program [38] from the training set and testing set respectively. The 27 selected testing sequences were summarized in Table S3 of the supplementary file.

(6) 21 sequences from 3'-UTRdb, whose second structure could not be predicted by RNAfold [39] or

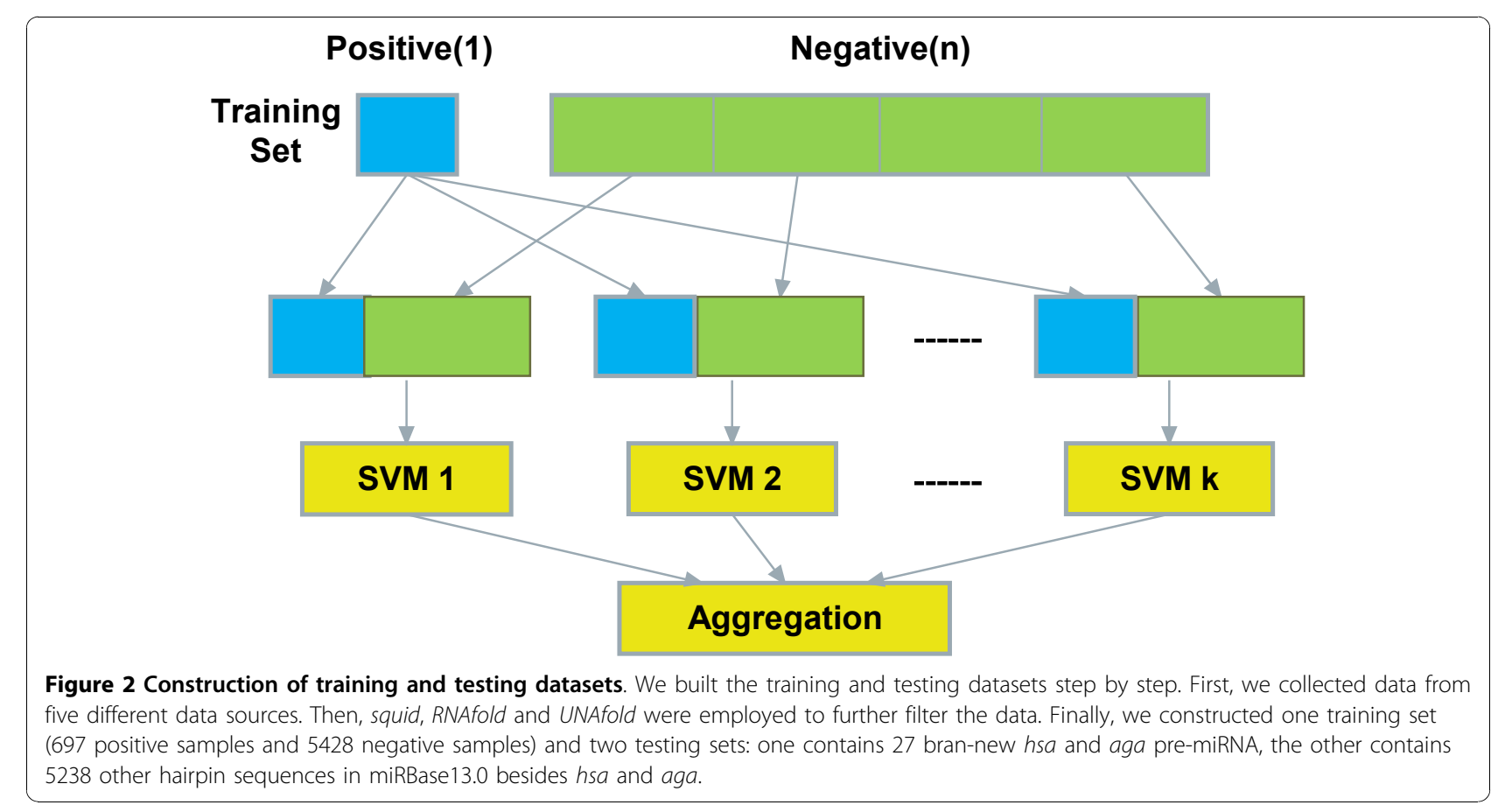


UNAfold [40] were removed. Finally, we constructed a training set with 697 true pre-miRNA sequences, 5428 pseudo pre-miRNA sequences, and a testing set with 27 bran-new real pre-miRNA sequences. After predicting the secondary structure, nearly $84 \%$ of the 5428 pseudo miRNA precursors have the secondary structure with multi-loop.

(7) 27 animal genomes (not including has and aga) in miRBase13.0 contain 5238 pre-miRNA sequences. We collected these sequences and used them to further evaluate the proposed approach miRenSVM.

\section{Feature selection}

The extraction of an appropriate set of features with which a classifier is trained is one of the most challenging issues in machine learning-based classifier development. In our study, both hairpin secondary structure and multi-loop structure features were considered. Concretely, we characterized a miRNA precursor by 65 local and global features that capture its sequence, secondary structure and thermodynamic properties. These features were subsumed into three groups as follows.

\section{2 triplet elements}

Sequence and structure properties are characterized by triplet structure-sequence elements proposed in [25]. In the predicted secondary structure, there are only two states for each nucleotide, paired or unpaired, indicated by brackets ('(' or or)') and dots ('.'), respectively. We do not distinguish these two situations in this work and use '(' for both situations, and GU wobble pair is allowed here. For any 3 adjacent nucleotides, there are 8 possible structure compositions: '(((', ‘((.', '...', ‘.(', ‘.((', ‘.(.', '...' and '...'. Considering the middle nucleotide among the 3 , there are $32\left(8^{*} 4\right)$ possible structure-sequence combinations, which are denoted as " $\mathrm{U}((($ ", "A $(()$, etc.

\section{5 base pair features}

Some secondary structure relevant features are already introduced by existing pre-miRNA classification methods $[27,32]$. In this paper, we included 11 secondary structure features $\left(G / C\right.$ ratio, $\% C+G, d P, A v g \_B P \_S t e m$, Diversity, $|A-U| / L,|G-C| / L,|G-U| / L,(A-U) / n \_$stems, (G$\left.C) / n \_s t e m s,(G-U) / n \_s t e m s\right)$ in our miRenSVM. Furthermore, for identifying real miRNA precursors with multiloop, we used four new features related to the loop number in the predicted secondary structure. They are:

- $d P / n_{-}$loops, where $n_{-}$loops is the number of loops in secondary structure.

$\%(A-U) / n_{-}$loops, $\%(G-C) / n_{-}$loops, $\%(G-U) / n_{-}$loops, where $\%(X-Y)$ is the ratio of $X-Y$ base pairs in the secondary structure.

These features were extracted using the RNAfold program contained in Vienna RNA package (1.8.3) [39] with default parameters.

\section{8 thermodynamic features}

It has been proved that using only secondary structure is not enough to effectively predict miRNA [30]. Since miRNA precursors usually have lower MFE than other small ncRNAs and random short sequences, thus MFE related features were introduced, such as $\left(d G, M F E I_{1}\right.$, $M F E I_{2}, M F E I_{3}, M F E I_{4}$, Freq). Other 8 global thermodynamics features (NEFE, Diff, $d H, d S, T m, d H / L, d S / L$, $T m / L$ ), and 4 statistically significant features ( $p$ value_MFE, $p$-value_EFE, $z$-score_MFE, $z$-score_EFE) were chosen from previous research $[23,24,36]$. When evaluating those statistically significant features related with MFE and ensemble free energy (EFE), for each original sequence, 300 random sequences were generated by Sean Eddy's squid program [30]. $d H, d S, T m, d H / L$, $d S / L, T m / L$ were calculated by UNAfold 3.7. More detail of all the 65 features are provided in additional file 1.

We used $F$-score to measure the discriminatory power of each feature above. $F$-score is a simple technique that measures the discrimination of two sets of real numbers. Given a set of training vectors $x_{k}, k=1, \ldots, m$, if the number of positive and negative instances are $n+$ and $n$, respectively, then the $F$-score of the $i$ th feature is defined as:

$$
F(i) \equiv \frac{\left(\bar{x}_{i}^{(+)}-\bar{x}_{i}\right)^{2}+\left(\bar{x}_{i}^{(-)}-\bar{x}_{i}\right)^{2}}{\frac{1}{n_{+}-1} \sum_{k=1}^{n_{+}}\left(x_{k, i}^{(+)}-\bar{x}_{i}^{(+)}\right)^{2}+\frac{1}{n_{-}-1} \sum_{k=1}^{n_{-}}\left(c_{k, i}^{(-)}-\bar{x}_{i}^{(-)}\right)^{2}}
$$

where $\bar{x}_{i}, \bar{x}_{i}^{(+)}, \bar{x}_{i}^{(-)}$are the average values of the $i$ th features of the whole, positive, and negative data sets, respectively; ${ }_{x, i}^{-+}$is the $i$ th feature of the $k$ th positive instance, and $x_{k, i}^{-(-)}$is the $i$ th feature of the $k$ th negative instance. Larger $F$-scores indicate better discrimination [41]. All the 65 local and global candidate features were ranked by $F$-score in order to determine which features will be used in the final model.

\section{The miRenSVM approach \\ Support vector machine}

The internal of miRenSVM is Support Vector Machine, a supervised classification technique derived from the statistical learning theory of structural risk minimization principle [42]. A support vector machine constructs a hyperplane or set of hyperplanes in a high-dimensional space, which can be used for classification, regression or other tasks. SVM has been adopted extensively as an effective discriminative machine learning tool to address the miRNA prediction problem [25, 27, 43]. The model selection for SVMs involves the selection of a kernel function and its parameters that yield the optimal 


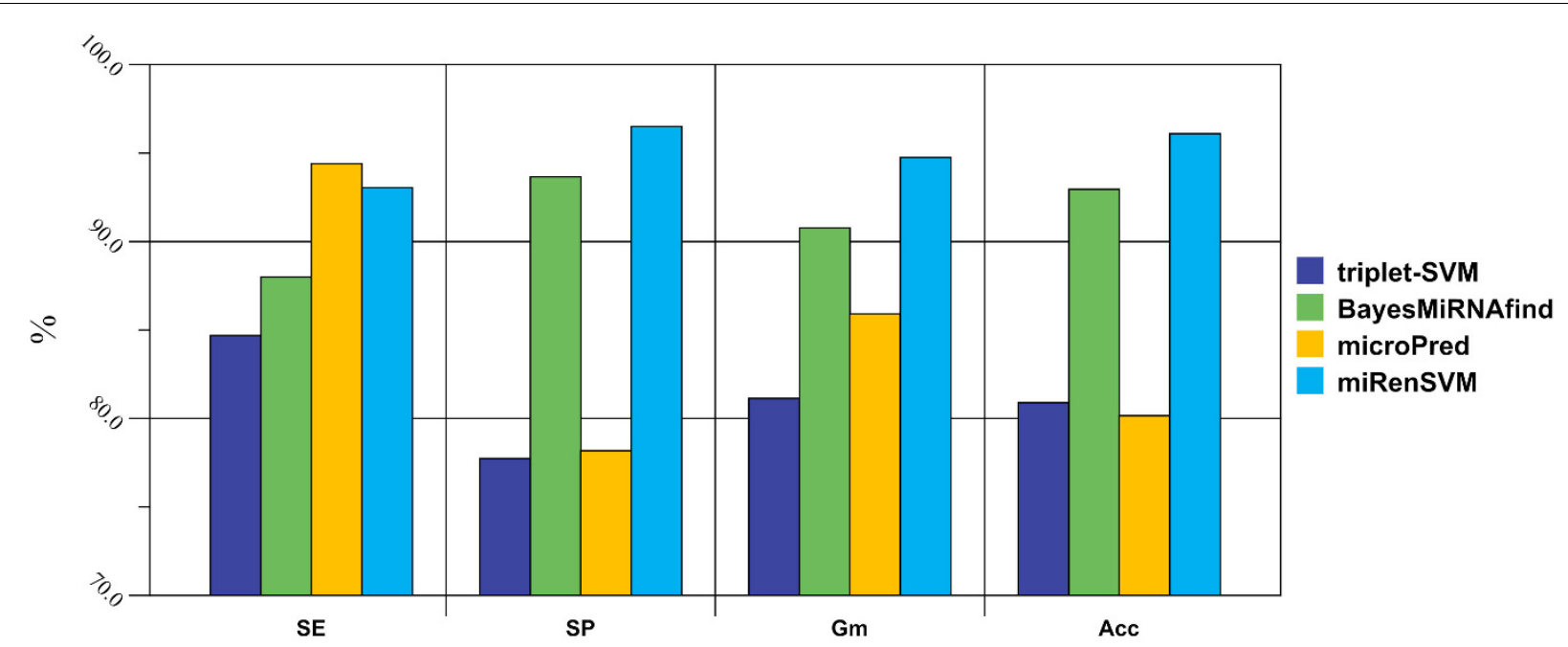

Figure 3 The Architecture of miRenSVM. The original negative samples in the training set are divided into $k$ equal partitions ( $k$ ranges from 1 to the ratio of negative samples to positive samples). The final decision is made by aggregating the results of $k$ sub-SVM classifiers that are trained by the entire positive samples and a partition of negative samples. Two aggregation methods are considered in this work: majority vote and mean distance.

classification performance for a given dataset [44]. In our study, we used radial basic function (RBF) due to its higher reliability in finding optimal classification solutions in most situations. The SVM algorithm was implemented by $\mathrm{C}++$ interface libsvm (version 2.89) package [41], and the training process of miRenSVM follows the guidelines described in [45].

\section{SVM classifiers ensemble}

One major factor that will influence the performance of a machine learning system is class imbalance, that is, the examples of some classes heavily outnumber the examples of the other classes [46]. Training a classifier system with an imbalance dataset will result in poor classification performance, especially for the rare classes [47]. And a classifier should have good and balanced performance over all classes for it to be useful in realworld applications.

For miRNA gene detection, the imbalance issue was widely recognized [32]. Existing machine learning based methods either employ random under-sampling to choose a portion of representative examples or just ignore it. It has already shown that both random over-sampling and random under-sampling have some drawbacks. The former does not add any information in addition to incurring large amount of compitation cost, and the later actually misses information and thus leads to poor performance. There remains a challenge: for a given dataset, how to select an appropriate sampling proportion?

In this work, the training dataset contains 697 positive (real pre-miRNA) samples and 5428 negative (pseudo pre-miRNA) samples, the ratio of negative to positive is 7.79:1. To address the drawbacks of over-sampling and under-sampling, we employed a SVM ensemble scheme. We tried to generate training sets with a desired distribution such that neither removing any training sample nor increasing the training time. An ensemble SVM classifier has several advantages over the ordinary classifiers. First, an ensemble SVM classifier exploits the information of the entire dataset, while random undersampling uses only part of the dataset; On the other hand, it consumes less computation compared to random over-sampling. Second, an ensemble SVM classifier is able to overcome some drawbacks of a single classifier. With multi sub SVM classifiers, miRenSVM is more robust and expected to learn the exact parameters for a global optimum [42].

Figure 3 shows the ensemble scheme of miRenSVM. Here, we used the same strategies as in [48] to sample the training dataset. First, splitting the negative examples into $k$ partitions where $k$ is chosen from 1 to the ratio of the majority class' size to the minority class' size. Second, generating individual subsets by combining the positive set with each partition of the negative samples. Third, training SVMs independently over every subset of the training set, and finally combining all constituent SVMs by certain of strategy to get the ensemble classifier. Majority vote is a widely used method to combine the results of several SVM sub-classifiers. In this paper, in addition to majority vote, we also used another technique called mean distance. Unlike majority vote, in the mean distance scheme, each sample is tested only one time by using one SVM subclassifier. While training classifiers, we evaluated the center vector of each training set. To classify an unlabeled sample, the distance between the sample and each center 
vector will be calculated, and the sample will be labelled by the SVM sub-classifier whose center vector is the nearest one to the sample under testing.

\section{Performance evaluation method and metrics Outer 3-fold cross validation}

We used the libsvm 2.89 package to establish the miRenSVM classification system. Here, the complete training dataset is randomly divided into three equally sized partitions, while each partition has the same ratio of positive samples to negative samples. Then, any two partitions are merged together as the training dataset to train an SVM classifier. Following that, the resulting model is tested over the third data partition. This procedure is repeated three times with different combinations of training (two partitions) and testing (the remaining partition) datasets in an outer 3 -fold cross validation style, and the classification result is gotten by averaging the results of the three tests above.

A straightforward way to evaluating the performance of a classifier is based on the confusion matrix. With this matrix, it is possible to evaluate a number of widely used metrics to measure the performance of a learning system, such as accuracy (Acc). However, Acc cannot be used to measure the performance of a classifier precisely when the class imbalance problem is present, as it does not reveal the true classification performance of the rare classes $[47,49]$. Therefore, in addition to Acc, we also used sensitivity (SE), specificity (SP) to evaluate the performance of a classifier. In order to exploit both positive and negative samples as much as possible, we also used their geometric mean $(\mathrm{Gm})$. Actually we pay more attention to $G \mathrm{~m}$ than to other three metrics, as $G \mathrm{~m}$ is an aggregated performance measure. These performance metrics are defined as follows:

$$
A c c=\frac{T P+T N}{T P+F P+T N+F N}, S E=\frac{T P}{T P+F N}, S P=\frac{T N}{T N+F P}, G_{m}=\sqrt{S E \square S P}
$$

where $T P, F P, T N$ and $F N$ are the numbers of true positive predictions, false positive predictions, true negative predictions and false negative predictions, respectively.

Additional file 1: Description: We surveyed pre-miRNA registered in miRBase with secondary multi-loop brunch secondary structure or with a MFE higher than $-16 \mathrm{kal} / \mathrm{mol}$, and showed the results in Table S1 and S2. Table S3 lists the 27 bran-new hsa and aga pre-miRNA sequences used as testing set. Table $\mathbf{S} 4$ shows the detail results of 27 other animal genomes. We also supplied some detail of the features used in the main paper

Additional file 2: Description: An Anopheles gambiae (aga) ncRNA dataset is built by selecting sequences whose secondary structures can be predicted by RNAfold and UNAfold in Rfam9.1. Furthermore, sequences with identity higher than $90 \%$ are removed.

\section{Acknowledgements}

The authors appreciate Prof. Malik Yousef and Mr. Manohara Rukshan Batuwita for predicting the test sequences.

This article has been published as part of BMC Bioinformatics Volume 11 Supplement 11, 2010: Proceedings of the 21st International Conference on Genome Informatics (GIW2010). The full contents of the supplement are available online at http://www.biomedcentral.com/1471-2105/11?issue=S11.

\section{Author details}

${ }^{1}$ Shanghai Key Lab of Intelligent Information Processing, and School of Computer Science, Fudan University, Shanghai 200433, China. ${ }^{2}$ Department of Computer Science and Technology, Tongji University, Shanghai 201804, China.

\section{Authors' contributions}

JD constructed the model, performed the experiments and prepared the manuscript. SG and JH guided the research and scheme design, and helped to prepare and improve the manuscript. All authors read and approved the manuscript.

\section{Competing interests}

The authors declare that they have no competing interests.

Funding: This research was supported by the National Basic Research

Program of China under grant no.2010CB126604.

Published: 14 December 2010

\section{References}

1. Lee RC, Feinbaum RL, Ambros V: The C. elegans heterochronic gene lin-4 encodes small RNAs with antisense complementarity to lin-14. Cell 1993, 75:843-54.

2. Bartel DP: MicroRNAs: genomics, biogenesis, mechanism, and function. Cell 2004, 116:281-97.

3. Ding XC, Weiler J, Grosshans H: Regulating the regulators: mechanisms controlling the maturation of microRNAs. Trends in biotechnology 2009, 27:27-36.

4. Chang T, Mendell JT: microRNAs in vertebrate physiology and human disease. Annual review of genomics and human genetics 2007, 8:215-39.

5. Esquela-Kerscher A, Slack FJ: Oncomirs - microRNAs with a role in cancer. Nature reviews. Cancer 2006, 6:259-69.

6. Lim LP, Lau NC, Weinstein EG, et al: The microRNAs of Caenorhabditis elegans. Genes \& development 2003, 17:991-1008.

7. Rodriguez A, Griffiths-Jones S, Ashurst JL, Bradley A: Identification of mammalian microRNA host genes and transcription units. Genome research 2004, 14:1902-10.

8. Smalheiser NR, Torvik VI: Mammalian microRNAs derived from genomic repeats. Trends in genetics : TIG 2005, 21:322-6.

9. Zhang $H$, Kolb FA, Jaskiewicz L, Westhof E, Filipowicz W: Single processing center models for human Dicer and bacterial RNase III. Cell 2004, 118:57-68.

10. Ghildiyal M, Xu J, Seitz H, Weng Z, Zamore PD: Sorting of Drosophila small silencing RNAs partitions microRNA* strands into the RNA interference pathway. RNA 2010, 16:43-56.

11. Okamura K, Liu N, Lai EC: Distinct mechanisms for microRNA strand selection by Drosophila Argonautes. Molecular cell 2009, 36:431-44.

12. Ambros V: microRNAs: tiny regulators with great potential. Cell 2001, 107:823-6.

13. Liang $H$, Li W: Lowly expressed human microRNA genes evolve rapidly. Molecular biology and evolution 2009, 26:1195-8.

14. Friedländer MR, Chen W, Adamidi $C$, et al: Discovering microRNAs from deep sequencing data using miRDeep. Nature biotechnology 2008, 26:407-15.

15. Grad $Y$, Aach J, Hayes GD, et al: Computational and experimental identification of C. elegans microRNAs. Molecular cell 2003, 11:1253-63.

16. Gautheret D, Lambert A: Direct RNA motif definition and identification from multiple sequence alignments using secondary structure profiles. Journal of molecular biology 2001, 313:1003-11.

17. Wang $X$, Zhang J, Li F, et al: MicroRNA identification based on sequence and structure alignment. Bioinformatics 2005, 21:3610-4. 
18. Bentwich I, Avniel A, Karov Y, et al: Identification of hundreds of conserved and nonconserved human microRNAs. Nature genetics 2005, 37:766-70.

19. Mendes ND, Freitas AT, Sagot M: Current tools for the identification of miRNA genes and their targets. Nucleic acids research 2009, 37:2419-33.

20. Nam J, Shin K, Han J, et al: Human microRNA prediction through a probabilistic co-learning model of sequence and structure. Nucleic acids research 2005, 33:3570-81.

21. Terai G, Komori T, Asai K, Kin T: miRRim: A novel system to find conserved miRNAs with high sensitivity and specificity. RNA 2007, 2081-2090.

22. Jiang $P, W u H$, Wang $W$, et al: MiPred: classification of real and pseudo microRNA precursors using random forest prediction model with combined features. Nucleic acids research 2007, 35:W339-44.

23. Yousef $M$, Nebozhyn $M$, Shatkay $H$, et al: Combining multi-species genomic data for microRNA identification using a Naive Bayes classifier. Bioinformatics 2006, 22:1325-1334.

24. $X u Y$, Zhou $X$, Zhang W: MicroRNA prediction with a novel ranking algorithm based on random walks. Bioinformatics 2008, 24:150-8.

25. Xue C, Li F, He T, et al: Classification of real and pseudo microRNA precursors using local structure-sequence features and support vector machine. BMC bioinformatics 2005, 6:310.

26. Hertel J, Stadler PF: Hairpins in a Haystack: recognizing microRNA precursors in comparative genomics data. Bioinformatics 2006, 22: e197-202.

27. Ng KL, Mishra SK: De novo SVM classification of precursor microRNAs from genomic pseudo hairpins using global and intrinsic folding measures. Bioinformatics 2007, 23:1321-30.

28. Huang T, Fan B, Rothschild MF, et al: MiRFinder: an improved approach and software implementation for genome-wide fast microRNA precursor scans. BMC bioinformatics 2007, 8:341

29. Griffiths-Jones S, Saini HK, van Dongen S, Enright AJ: miRBase: tools for microRNA genomics. Nucleic acids research 2008, 36:D154-8.

30. Rivas E, Eddy SR: Secondary structure alone is generally not statistically significant for the detection of noncoding RNAs. Bioinformatics 2000, 16:583-605.

31. Singhi SK, Liu H: Feature subset selection bias for classification learning. Proceedings of the 23rd international conference on Machine learning - ICML '06 2006, 849-856.

32. Batuwita R, Palade V: microPred: effective classification of pre-miRNAs for human miRNA gene prediction. Bioinformatics 2009, 25:989-95.

33. Scott MS, Avolio F, Ono M, Lamond Al, Barton GJ: Human miRNA precursors with box H/ACA snoRNA features. PLOS computational biology 2009, 5:e1000507.

34. Pesole G, Liuni S, Grillo G, et al: UTRdb and UTRsite: specialized databases of sequences and functional elements of $5^{\prime}$ and $3^{\prime}$ untranslated regions of eukaryotic mRNAs. Update 2002. Nucleic acids research 2002, 30:335-40.

35. Gardner PP, Daub J, Tate JG, et al: Rfam: updates to the RNA families database. Nucleic acids research 2009, 37:D136-40.

36. Chatterjee $\mathrm{R}$, Chaudhuri $\mathrm{K}$ : An approach for the identification of microRNA with an application to Anopheles gambiae. ACTA BIOCHIMICA POLONICA-ENGLISH 2006, 53:303-309.

37. Griffiths-Jones S: Annotating noncoding RNA genes. Annual review of genomics and human genetics 2007, 8:279-98.

38. Li W, Godzik A: Cd-hit: a fast program for clustering and comparing large sets of protein or nucleotide sequences. Bioinformatics 2006, 22:1658-9.

39. Hofacker IL: Vienna RNA secondary structure server. Nucleic acids research 2003, 31:3429-31.

40. Markham NR, Zuker M: DINAMelt web server for nucleic acid melting prediction. Nucleic acids research 2005, 33:W577-81.

41. Chen Yi-Wei, Lin Chih-Jen: Combining SVMs with Various Feature Selection Strategies. Studies in Fuzziness and Soft Computing Springer Berlin / Heidelberg; 2006, 10.

42. Burges C: A tutorial on support vector machines for pattern recognition. Data mining and knowledge discovery 1998, 2:121-167.

43. Sheng $Y$, Engström PG, Lenhard B: Mammalian microRNA prediction through a support vector machine model of sequence and structure. Plos one 2007, 2:e946.

44. Duan K: Evaluation of simple performance measures for tuning SVM hyperparameters. Neurocomputing 2003, 51:41-59.

45. Hsu C, Chang C: A practical guide to support vector classification. Bioinformatics 2003, 1:1-16.
46. Batista GE, Prati RC, Monard MC: A study of the behavior of several methods for balancing machine learning training data. ACM SIGKDD Explorations Newsletter 2004, 6:20.

47. Weiss GM: Mining with rarity. ACM SIGKDD Explorations Newsletter 2004, 6:7.

48. Rong Yan, Yan Liu, Rong Jin AH: On predicting rare classes with SVM ensembles in scene classification. 2003 IEEE International Conference on Acoustics, Speech, and Signal Processing, 2003. Proceedings. (ICASSP '03) 2003, 6-10.

49. Akbani R, Kwek S, Japkowicz N: Applying support vector machines to imbalanced datasets. Machine Learning: ECML 20042004.

doi:10.1186/1471-2105-11-S11-S11

Cite this article as: Ding et al:: MiRenSVM: towards better prediction of microRNA precursors using an ensemble SVM classifier with multi-loop features. BMC Bioinformatics 2010 11(Suppl 11):S11.

\section{Submit your next manuscript to BioMed Central and take full advantage of:}

- Convenient online submission

- Thorough peer review

- No space constraints or color figure charges

- Immediate publication on acceptance

- Inclusion in PubMed, CAS, Scopus and Google Scholar

- Research which is freely available for redistribution

Submit your manuscript at www.biomedcentral com/submit
C) Biomed Central 